\title{
Caractérisation des vagues dans les passes du bassin d'Arcachon
}

\author{
Rodrigo PEDREROS ${ }^{1}$, Sophie LECACHEUX ${ }^{1}$, Aldo SOTTOLICHIO ${ }^{2}$, \\ Emmanuel ROMIEU ${ }^{1}$, Déborah IDIER ${ }^{1}$, Paulo SALLES ${ }^{2,3}$, Matthieu \\ DELATTRE ${ }^{1}$
}

${ }^{1}$ BRGM, Service Aménagement et Risques Naturels

3 av. C. Guillemin, BP 6009, 45060 Orléans

r.pedreros@brgm.fr, s.lecacheux@brgm.fr, e.romieu@brgm.fr, $\underline{\text { d.idier@brgm.fr, }}$ m.delattre@brgm.fr

${ }^{2}$ Université de Bordeaux 1 - UMR 5805 EPOC - OASU

avenue des Facultés, 33405 Talence

a.sottolichio@epoc.u-bordeaux1.fr

${ }^{3}$ Universidad Autonoma de Mexico (UNAM),

Instituto de Ingenieria, 5-306, Mexico D.F., Mexique

\section{Résumé :}

psallesa@iingen.unam.mx

L'objectif de cette étude est d'identifier l'influence de l'hydrodynamique tidale et de la géométrie des passes sur la propagation de la houle dans les passes du bassin d'Arcachon. Deux courantomètres profileurs ont enregistré la hauteur d'eau, les courants et les vagues en janvier-février 2007. Une modélisation sur le mois de février 2007 a été effectuée avec les codes MARS 2DH, pour la marée et les courants de marée, et SWAN pour les vagues. Les résultats des premières simulations sont cohérents avec les mesures et montrent l'influence majeure de la hauteur d'eau et des courants de marée sur les variations de la hauteur significative des vagues. Le rôle de la configuration des passes et des bancs dans l'hydrodynamique du milieu est mis en évidence.

\section{Abstract :}

This paper investigates the influence of the current and the tide on the wave propagation in the channels of the Arcachon basin. The study is based on twomonth hydrodynamic in-situ measurements and a 2DH model using MARS 2DH and SWAN codes. The first results show a good agreement between the results of the model and the measurements. The velocities and the wave propagation seem to be very sensitive to the topography of the channel and the sandbanks.

Mots-clés :

SWAN - MARS-2DH - Bassin d'Arcachon - Houle - Courant - Passes Modélisation - Bancs sableux 


\section{$1 \underline{\text { Introduction }}$}

Le bassin d'Arcachon est un site représentatif des systèmes semi fermés. Une embouchure tidale, composée de deux passes (MICHEL, 1997), constitue le seul lien entre l'océan et le bassin peu profond (Figure 1). Séparées par le banc d'Arguin, les passes sont en évolution permanente à cause d'une dynamique forte (courants de marée et houle) qui induit un transport sédimentaire intense et une morphologie particulièrement active (GASSIAT, 1989). longitude $\left({ }^{\circ} \mathrm{E}\right)$

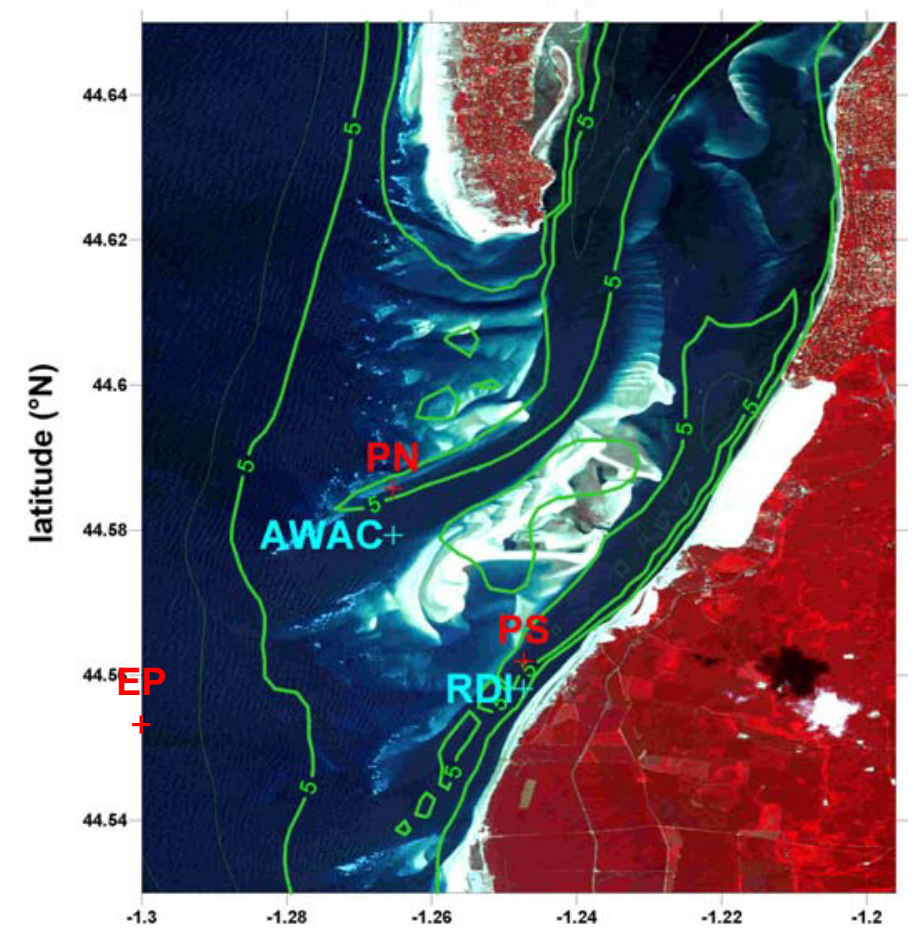

Figure 1. Configuration des passes et du bassin d'Arcachon (image SPOT 5 de juin 2004). Les contours verts représentent la cote du fond utilisée au dernier rang des simulations.

L'objectif de cette étude est d'identifier, comprendre et modéliser l'influence de l'hydrodynamique tidale et de la géométrie des passes et des bancs, sur la propagation de la houle dans les passes. C'est la première étape pour développer à terme un outil de modélisation morphodynamique de ce système.

Cette étude présente, dans un premier temps l'analyse des mesures de hauteur d'eau, de courants et de vagues. Ensuite, afin de tester les hypothèses émises, deux codes dédiés à l'hydrodynamique côtière sont utilisés pour calculer les champs de vagues (code SWAN) en prenant en compte l'influence de l'hydrodynamique tidale (code MARS-2DH). Il est à noter que la génération du 
clapot dans le bassin est traitée par PARISOT et al. (2008) dans le cadre de cette conférence.

\section{Mesures in-situ}

\subsection{La campagne}

Les mesures dans les passes ont été acquises lors d'une campagne réalisée du 15/01/07 au 04/03/07 (SALLES et al. 2008). Deux courantomètres profileurs à effet Doppler en mode vagues ont été utilisés : un AWAC $600 \mathrm{kHz}$ (de Nortek) et un ADCP $1200 \mathrm{kHz}$ (de RDI). Ils ont été positionnés respectivement dans la passe nord $\left(1,265^{\circ} \mathrm{W} / 44,579^{\circ} \mathrm{N}\right.$ à $6 \mathrm{~m}$ de fond cote marine- $\left.\mathrm{CM}\right)$ et dans la passe sud $\left(1,247^{\circ} \mathrm{W} / 44,558^{\circ} \mathrm{N}\right.$ à $19 \mathrm{~m}$ de fond $\left.\mathrm{CM}\right)$, et ont enregistré le niveau d'eau, les courants et les vagues (Figure 1). A ce jour, seules les données de l'ADCP dans la passe sud ont été analysées et seront présentées. Les tendances générales des courants bruts et des vagues mesurés par l'AWAC seront seulement évoquées (elles seront approfondies lors de la présentation).

Les mesures de hauteur d'eau et de courant sont traitées pour distinguer le signal de marée (analyse des composantes harmoniques avec le logiciel T-Tide, PAWLOWICZ et al. 2002) et le signal résiduel (IDIER et al., 2006). De plus, pour comparer directement ces données traitées aux résultats du modèle, les courants mesurés par les profileurs sont intégrés sur la verticale. Quant aux vagues, elles sont traitées avec le logiciel WavesMon de RDI (Figure 2).

\subsection{Hauteur d'eau et courants}

On note Figure 2 (C et D), que la marée (niveau d'eau et courant) est le phénomène dominant jusqu'au 7 février: les surcotes sont négligeables et les courants bruts sont très proches des courants de marée.

La marée (Figure 2, C) a un marnage d'environ $1 \mathrm{~m}$ en mortes-eaux et atteint $4 \mathrm{~m}$ en vives-eaux.

Dans la passe sud, les courants de marée (Figure 2, D) atteignent 1,2 m/s à $1,4 \mathrm{~m} / \mathrm{s}$ en vives-eaux (le 05 et $19 / 02$ ) et $0,6 \mathrm{~m} / \mathrm{s}$ en mortes-eaux $(11 / 02)$. En mortes-eaux, le courant de jusant est plus long et un peu plus fort que le courant de flot. En vives-eaux, les deux courants sont à peu près équivalents en durée et en intensité.

Dans la passe nord, une première visualisation des données brutes (SALLES 2008, com. personnelle) montre que le courant de jusant est toujours plus intense que le courant de flot. Les courants de marée sont globalement plus forts que dans la passe sud. En vives-eaux, les courants de jusant atteignent $2 \mathrm{~m} / \mathrm{s}$ et ceux de flot avoisinent $1 \mathrm{~m} / \mathrm{s}$. 


\subsection{Les vagues}

La Figure 2 (A, B et E) montre clairement que les conditions de vent et de vagues au large ont peu d'influence sur les hauteurs des vagues au niveau de la passe sud. Les valeurs les plus importantes de $0,8 \mathrm{~m}$ à $0,95 \mathrm{~m}$ ont lieu le 7 et du 14 au 23 février. Elles ne correspondent pas à la période la plus énergétique du 9 au 13 février. En effet, cette période coïncide avec les mortes-eaux. La hauteur de la marée apparaît donc comme un facteur prépondérant. En mortes-eaux la hauteur des vagues au niveau de la passe sud n'excède pas $0,65 \mathrm{~m}$ et cela pour des vagues au large allant jusqu'à $9 \mathrm{~m}$ de hauteur. En vives-eaux cette valeur seuil atteint $0,95 \mathrm{~m}$ pour une hauteur significative maximale au large mesurée à $7 \mathrm{~m}$.

La hauteur des vagues varie également avec la marée semi diurne (Figure 2, C, E et F). Par exemple, le matin du 5 en vives-eaux, Hs passe de $0,4 \mathrm{~m}$ (pleine mer) à $0,1 \mathrm{~m}$ (basse mer). En mortes-eaux, Hs varie moins, les valeurs se situent entre $0,3 \mathrm{~m}$ et $0,5 \mathrm{~m}$. D'une façon générale, la hauteur des vagues à marée basse ne dépasse pas $0,30 \mathrm{~m}$ et cela quelque soit le forçage externe ou le coefficient de marée considéré.

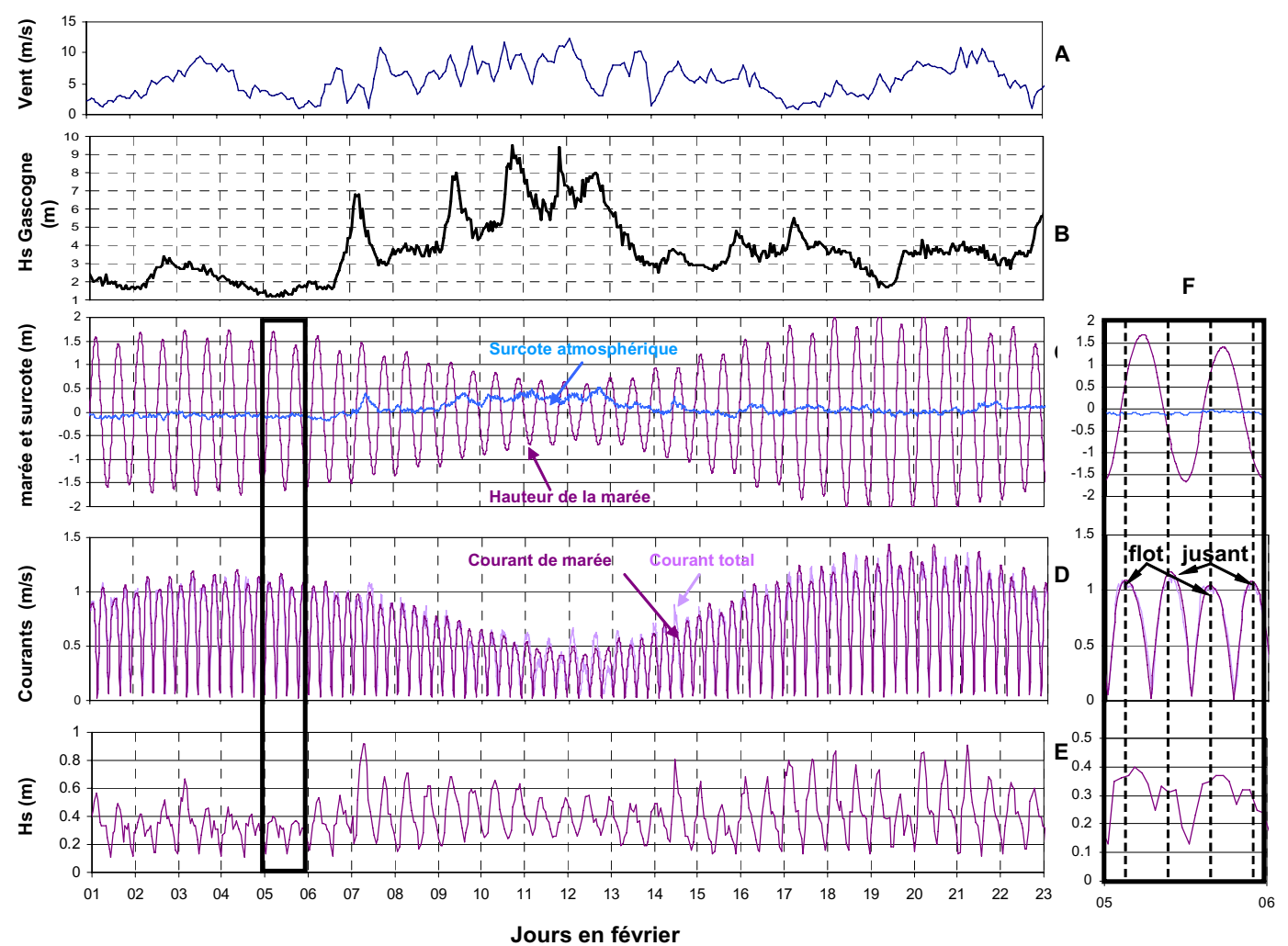

Figure 2. A : intensité du vent GFS. B : Hs mesuré par la bouée Gascogne $\left(45,20^{\circ} \mathrm{N} 5,00^{\circ} \mathrm{W}\right) . C$ à $F$ : mesures de l'ADCP RDI passe sud. $C$ : hauteur d'eau. D : courants et $E$ : Hs. F : zoom des mesures journée du 5 février. 
Les courants de marée (Figure 2, D) ont aussi une influence sur la hauteur des vagues, notamment en vives-eaux. Par exemple, le 5 février (Figure $2 \mathrm{~F}$ ), on remarque que le courant de flot induit une diminution de la hauteur des vagues (environ $0,05 \mathrm{~m}$ ) qui augmente ensuite sous l'action du courant de jusant (jusqu'à $0,08 \mathrm{~m})$.

On retrouve cette modulation tidale de la hauteur des vagues sur le capteur mouillé dans la passe nord, néanmoins les hauteurs des vagues enregistrées sont supérieures à celles de la passe sud. Hs atteint au maximum 2,5 $\mathrm{m}$ à marée haute et $0,8 \mathrm{~m}$ à marée basse le 7 février (SALLES 2008, com. Personnelle).

\section{Modélisation}

\subsection{Description et mise en place des modèles}

La modélisation a été effectuée du $1^{\text {er }}$ février 2007 au 23 février 2007.

Tout d'abord, la marée et les courants de marée sont calculés avec le code MARS 2DH développé par l'Ifremer. Une technique de modélisation emboitée avec 3 rangs est utilisée (IDIER, 2007). La boîte de grande emprise R0 couvre 1'Atlantique NW, puis les boîtes R1 et R2 sont centrées sur le bassin d'Arcachon. La grille finale, $\mathrm{R} 2$, a une résolution de $235 \mathrm{~m}$. Elle s'étend de $1,45^{\circ} \mathrm{W}$ à $0,95^{\circ} \mathrm{W}$ et de $44,4^{\circ} \mathrm{N}$ à $44,9^{\circ} \mathrm{N}$. Les conditions aux limites (signal de marée) sont issues de la base de données FES2004 (LYARD et al. 2006).

Puis, les vagues sont calculées avec le code SWAN de l'université de Delft (BOOIJ et al. 2004), en mode non stationnaire avec un pas de temps horaire, et prise en compte des hauteurs d'eau et courants calculés par le code MARS. Un modèle emboîté avec 2 rangs est utilisé. La première grille R1 couvre l'ensemble de la côte aquitaine. La frontière ouest de la grille est forcée avec les conditions de houle (hauteur significative, période de pic, direction de pic) issues du modèle WaveWatch 3 de la NOAA. Le vent, appliqué sur toute la grille, provient du modèle GFS également opéré par la NOAA (résolution spatiale de $0,5^{\circ} \times 0,5^{\circ}$ et selon une fréquence de 3 heures). La grille R2 est la même que celle utilisée par MARS 2DH. Les conditions de houle aux bords sont issues du calcul sur R1. Le vent (GFS) est appliqué sur toute la grille.

Les données bathymétriques utilisées dans le modèle sont antérieures à 2006 . Des différences entre la bathymétrie utilisée et la configuration réelle lors de la campagne des mesures ont été constatées grâce à une image satellite (Figure 1) et aux hauteurs d'eau mesurées par les instruments. La passe sud était notamment plus profonde, plus étroite et plus longue.

L'analyse des résultats des modèles dans les passes est réalisée avec deux capteurs fictifs correspondant au mieux aux positions des instruments de mesure 
(Figure 1) : le premier dans la passe nord, noté PN (positionné à 5,47 $\mathrm{m}$ de fond $\mathrm{CM}$ ) et le deuxième dans la passe sud, noté PS (positionné à $7 \mathrm{~m}$ de fond $\mathrm{CM}$ ). Le point de référence à l'entrée des passes à $20 \mathrm{~m}$ de profondeur est noté EP.

\subsection{Marée et courants de marée}

La marée (Figure 3, A et B) est bien représentée avec une erreur maximale de $20 \mathrm{~cm}$ à marée haute en vives-eaux.

Dans la passe sud, le modèle surestime fortement le courant de jusant, surtout en vives-eaux (Figure 3, D). En effet, la différence d'intensité entre les courants de flot et jusant calculés est de $0,8 \mathrm{~m} / \mathrm{s}$ alors que les mesures indiquent des courants d'intensités similaires. En mortes-eaux (Figure 3, C), les courants calculés sont plus en accord avec les mesures.

Dans la passe nord, les courants modélisés sont plus intenses que dans la passe sud, surtout le courant de jusant. En vives-eaux, les courants de jusant sont de l'ordre de $2 \mathrm{~m} / \mathrm{s}$ et ceux de jusant de $1,1 \mathrm{~m} / \mathrm{s}$. Ceci correspond bien aux tendances observées dans la première visualisation des données brutes de l'AWAC.
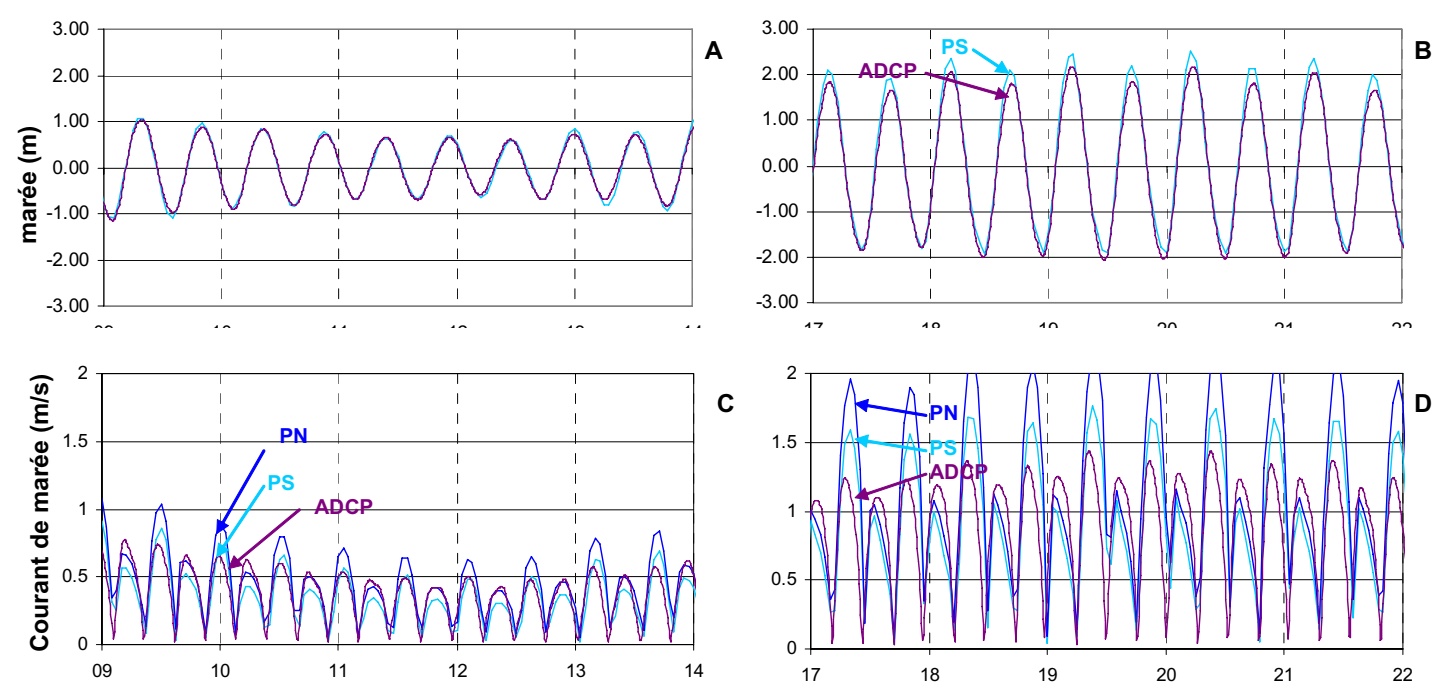

Figure 3. Résultats de la simulation de MARS 2DH et comparaison avec les mesures de l'ADCP RDI. A : marée, $B$ et $C$ : courants de marée respectivement en vives-eaux et en mortes-eaux.

Au moment de la campagne de mesures, la passe sud était plus étroite, allongée et profonde que la représentation de la bathymétrie utilisée. Le fait d'utiliser une bathymétrie trop éloignée de l'état réel des fonds pendant la campagne de mesure pourrait expliquer les écarts entre modèle et mesure dans cette zone. 


\subsection{Vagues}

\subsubsection{Influence du forçage au large et de l'hydrodynamique tidale}

La modélisation permet de bien retrouver les tendances issues de l'analyse des mesures (Figure $4 \mathrm{~A}$ et $\mathrm{B}$ ). On retrouve la faible dépendance de la hauteur des vagues par rapport au forçage externe. Les ordres de grandeurs sont respectés pour la passe nord. Pour la passe sud les valeurs sont trop importantes. Le maximum modélisé est de $1,7 \mathrm{~m}$ alors que la valeur observée est de $0,95 \mathrm{~m}$.

. Les variations de Hs dues à la marée semi diurne apparaissent également dans les deux passes. En vives-eaux, le 21/02/07, les vagues font près de 1,7 $\mathrm{m}$ dans la passe sud à marée haute alors qu'elles ne dépassent pas les $0,45 \mathrm{~m}$ à marée basse.
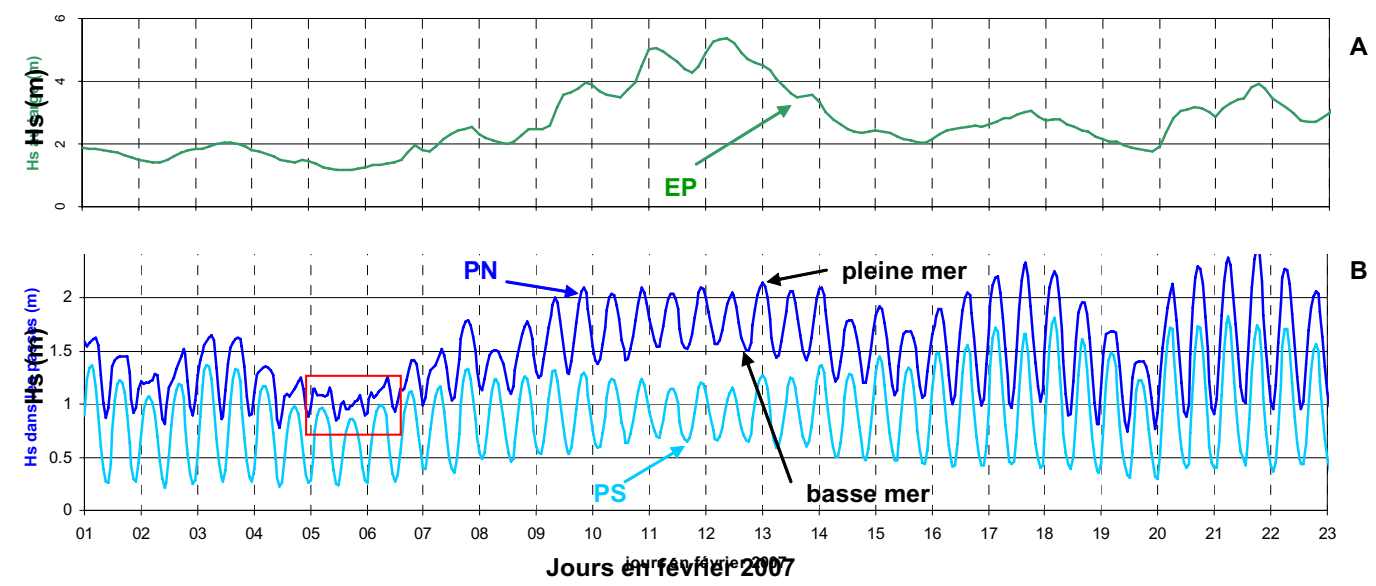

Figure 4. Hauteur significative des vagues calculée par SWAN.

L'influence du courant du jusant et de flot apparaît de manière claire dans la modélisation des vagues en mortes-eaux et en vives-eaux (Figure 5). L'impact est faible en mortes-eaux (courants peu intenses). En vives-eaux, le courant de jusant accentue la hauteur des vagues et favorise leur pénétration vers les passes internes. Cette tendance est inversée en cas de courant de flot intense.

On remarque Figure 5 que le banc d'Arguin est systématiquement submergé, quelque soit la marée, car son altitude est nulle dans la bathymétrie utilisée. De plus, son emprise est plus faible que sur l'image satellite (Figure 1). En conséquence, les vagues déferlent peu et ne sont presque pas dissipées dans ce secteur. Cela pourrait expliquer la surestimation de la hauteur significative calculée au point PS (Figure 4, B). 

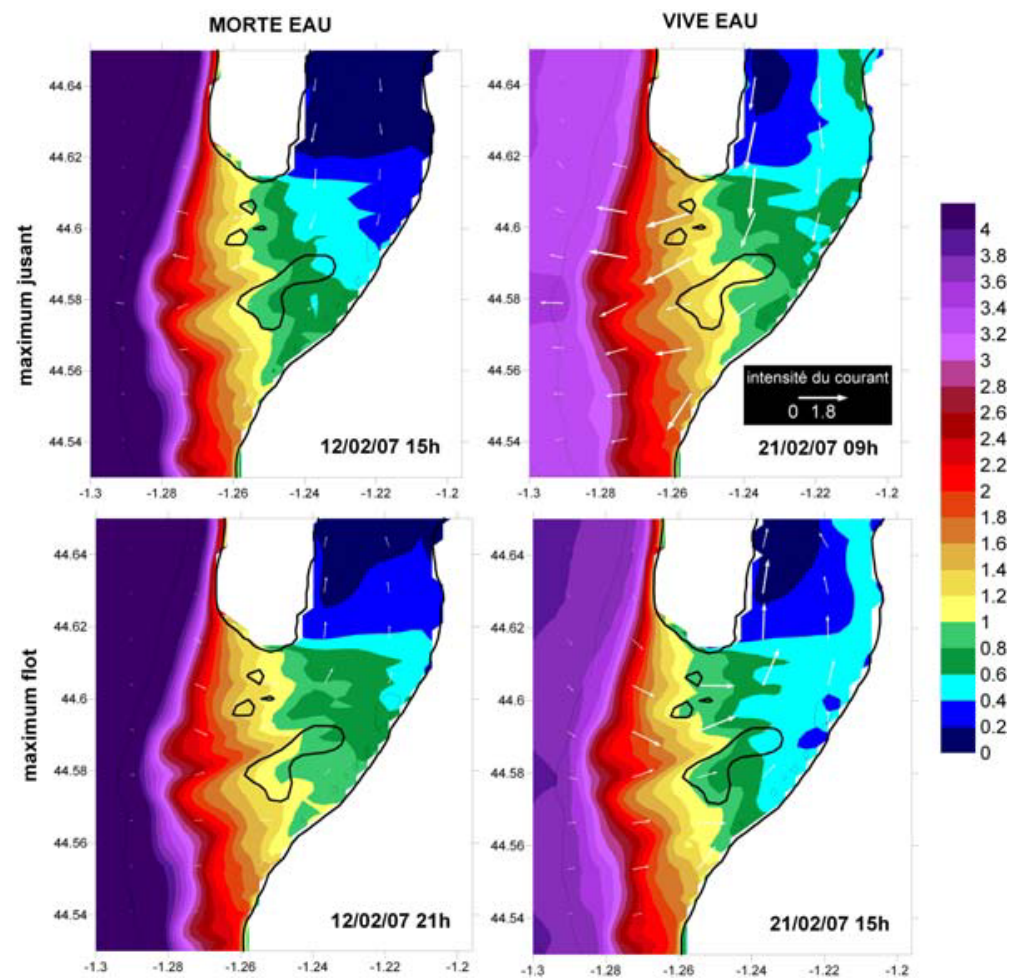

Figure 5. Hauteur significative des vagues dans les passes et le bassin d'Arcachon pour une houle $4 \mathrm{~m}$ au large et un vent d'ouest de $10 \mathrm{~m} / \mathrm{s}$.
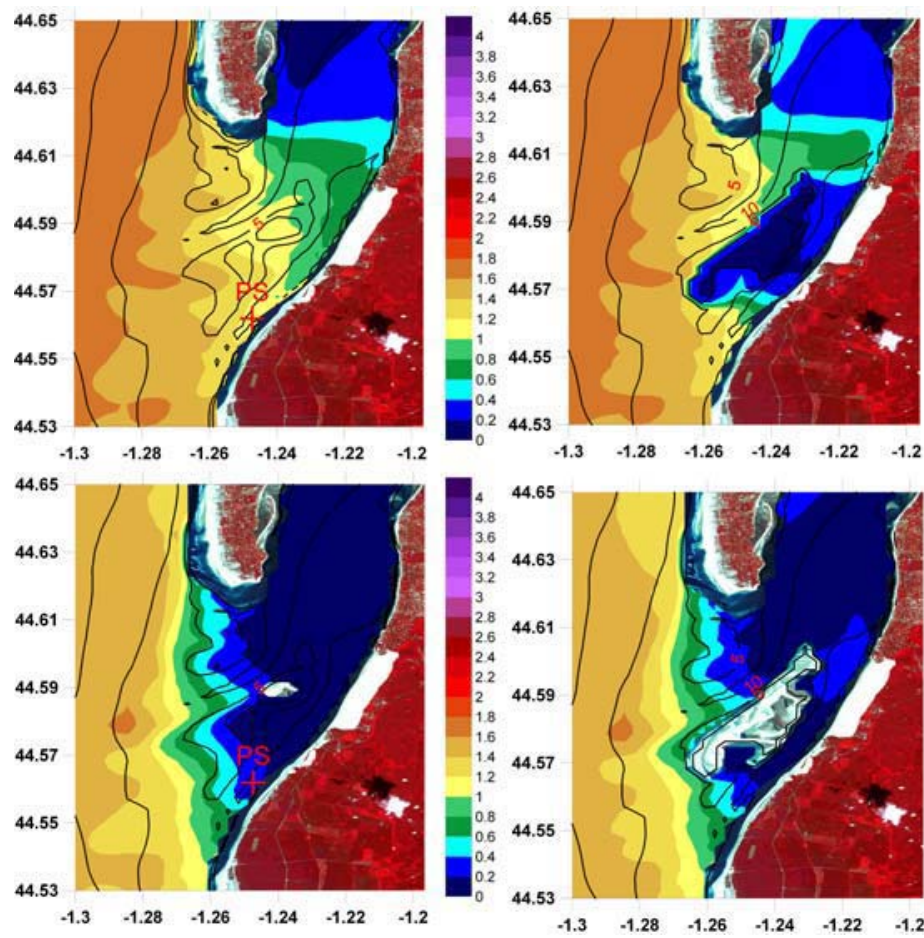

Figure 6. Hs à l'entrée des passes le 04/02/07 à marée haute et à marée basse avec la bathymétrie initiale (à gauche) et la bathymétrie modifiée (à droite). 


\subsubsection{Influence de la configuration des passes}

Pour tester cette hypothèse, une nouvelle simulation est effectuée en modifiant la bathymétrie initiale afin de représenter de façon plus réaliste le banc d'Arguin (modification de ces limites et attribution d'une altitude de $4 \mathrm{~m}$, marnage maximum de février). La Figure 6 présente les résultats des calculs de Hs à marée basse et à marée haute en utilisant la bathymétrie initiale et la bathymétrie modifiée. On remarque qu'à marée basse, il y a peu de différence. L'influence se manifeste essentiellement à marée haute. La nouvelle configuration contribue au déferlement des vagues au voisinage du banc. La passe sud est alors protégée. Les nouvelles valeurs de Hs sont généralement plus faibles de $0,30 \mathrm{~m}$ à $0,50 \mathrm{~m}$ (à marée haute) se rapprochant ainsi des mesures effectuées.

\section{Conclusions et perspectives}

Les mesures effectuées montrent la complexité du fonctionnement hydrodynamique dans les passes du bassin d'Arcachon. Les conditions très énergétiques, les courants de marée intenses et la morphologie très active rendent cet environnement exigeant à modéliser.

Les premières simulations montrent que la hauteur des vagues varie fortement avec la marée semi diurne.

La modélisation de l'interaction houle-courant donne des résultats encourageants : l'influence des courants de flot et de jusant apparait dans les deux passes.

La configuration des passes et des bancs a peu d'impact sur l'onde de marée mais joue sur l'intensité des courants et plus encore sur la propagation des vagues. La forme du banc d'Arguin, notamment, contrôle la répartition des courants de marée dans les passes ainsi que le déferlement et la dissipation des vagues dans la passe sud. L'utilisation d'images satellite comme SPOT (LAFON et al. 2000) et de spatiocartes est une piste à envisager pour obtenir une bathymétrie plus réaliste.

\section{$5 \quad$ Références bibliographiques}

1 BOOIJ, N., HAAGSMA IJ.G.; HOLTHUIJSEN L.H.; KIEFTENBURG, A.T.M.M.; RIS, R.C.; VAN DER WESTHUYSEN A.J. and ZIJLEMA M., (2004). Swan Cycle III version 40.41. User's Manual, 115p.

2 GASSIAT, L., (1989). Hydrodynamique et evolution sédimentaire d'un système lagune-flèche littoral (Bassin d'Arcachon-Cap Ferret). Thèse de $3^{\text {ème }}$ cycle, Université Bordeaux I, 228p.

3 IDIER D. (2007). Previmer - Opérationnalisation de modèles hydrodynamiques régionaux - Délivrables D1: Réalisation et mise en 
œuvre opérationnelle des modèles hydrodynamiques régionaux Arcachon, Bretagne Nord et Normandie. Rapport BRGM/RP-55861-FR, 135 p., 66 fig, 29 tabl.

4 IDIER D., PEDREROS R., OLIVEROS C., SOTTOLICHIO A., CHOPPIN L. et BERTIN X., (2006). Contributions respectives des courants et de la houle dans la mobilité sédimentaire d'une plateforme interne estuarienne. Exemple : le seuil interinsulaire, au large du Pertuis d'Antioche, France. C .R. Geoscience, Vol. 338, 718-726.

5 LAFON V., FROIDEFOND JM., CASTING P., (2000). Methode d'analyse de l'évolution morphodynamique d'une embouchure tidale par imagerie satellite. Exemple du Bassin d'Arcachon (France), Comptes Rendus de l'Académie des Sciences de Paris, 331, 373-378.

6 LYARD F., F. LEFEVRE, T. LETELLIER and O. FRANCIS, (2006), Modelling the global ocean tides: modern insights from FES2004, Ocean Dynamics, DOI 10.1007/s10236-006-0086-x.

7 MICHEL, D., HOWA, H., (1997). Morphodynamic behavior of a tidal inlet system in a mixed-energy environment. Physics \& Chemistry of the Earth 22, 339-343.

8 PARISOT J-P., DIET-DAVANCENS J., SOTTOLICHIO A., CROSLAND E., DRILLON C, (2008). Modélisation des agitations dans le Bassin d'Arcachon. JNGCGC 2008.

9 PAWLOVICZ R., BEARDSLEY B. and LENTZ S., (2002). Classical Tidal Harmonic Analysis Including Error Estimates in MATLAB using T_TIDE, Computers and Geosciences, 28, 929-937.

10 SAlles, P., A. SOTTOLiCHIO, P. BRETEL, S. BUJAN and R. PEDREROS, (2008). Tidal Distortion in the Arcachon Basin. XI International Symposium on Oceanography of the Bay of Biscay, 2-4 April, San Sebastian (Spain), Book of Abstracts "Revista de Investigacion Marina", p161-162.

\section{Remerciements}

Cette étude s'inscrit dans le Programme National d'Environnement Côtier (PNEC) sur les modes de fonctionnement des systèmes semi fermés.

Les auteurs remercient l'Ifremer pour la mise à disposition des données bathymétriques et de la configuration de MARS 2DH sur le bassin d'Arcachon ainsi que RDI pour le prêt de l'ADCP. 